\title{
Relationship between stress, anxiety, depression and salivary $\lg A$ with periodontal disease
}

\author{
Mohammadreza Karimi ${ }^{1}$, Shahryay Elyahoo ${ }^{2 *}$, Leyla Golchin ${ }^{3}$ and Tahereh Kermani ${ }^{4}$ \\ ${ }^{1}$ Assistant Professor, Periodontics Department, Dental Branch, Islamic Azad University, Tehran, Iran \\ ${ }^{2}$ Post graduate student, Periodontics Department, Dental Branch, Islamic Azad University, Tehran, Iran \\ ${ }^{3}$ General Dentist \\ ${ }^{4}$ Psychiatrist
}

\begin{abstract}
Psychological stress, if sustained over an extended period of time can have deleterious effects on the body. Psychological stress has been implicated as risk indicators for periodontal disease. So, the aim of the current study was to determine the relationship of stress, anxiety and depression level and salivary IgA with periodontal disease. A total 30 patients who referred to periodontology included to the study and divided into 2 experimental groups. Group control patients do not suffer from any periodontal disease and patients CAL up 3 millimeters or more and BOP in upper teeth (case group). All cases were evaluated for stress, anxiety and depression level by DASS42 test. Salivary samples were obtained using spitting method and IgA level is determined with ELISA. Data was analyzed by $\kappa 2$ and Mendle-hazel tests. The patients suffering from periodontal disease were not encountering higher level of stress and anxiety $(\mathrm{P}=0.3)$. People suffering periodontal disease $(86.7 \%)$ were depressed while $60 \%$ of people neither periodontal disease nor depressed $(\mathrm{P}=0.1)$. A significant difference detected in salivary IgA level in control group (312.66+107.3) compared to case group $(207.95+57.21)(\mathrm{P}=0.001)$. Conclusion: these results suggested a correlation exists between incidence of the periodontal disease and immunity.
\end{abstract}

KEY WORDS: PERIODONTAL DISEASE, STRESS, ANXIETY, DEPRESSION, IGA

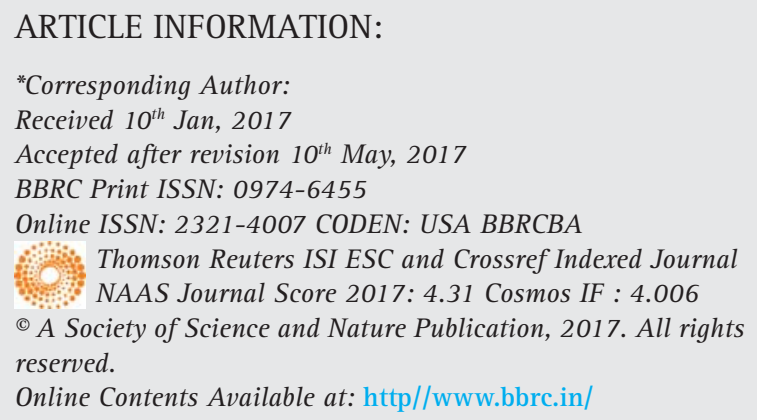




\section{INTRODUCTION}

Periodontal disease is a multifactorial disease. Dental plaque which harbours specific periodontal pathogens is its main aetiologic factor. Numerous factors have been associated with periodontitis, such as systemic diseases, genetic polymorphisms, socio-economic and/or educational status, smoking and psychological stress. Stress is considered as one of the essential factors with etiology of the periodontal disease (Boyapati and Wang, 2007). Psychological stress down regulates the cellular immune response (Castro et al. 2006). There is interconnection among the central nervous system and the immune system which happens via a complex network of bidirectional signals linking the nervous, endocrine, and immune systems (Gundala et al. 2012 Shende et al. (2016).

Chronic stress has a net negative effect on the immune response which leads to an imbalance between host and parasites and consequently resulting in periodontal break down (Radafshar et al. 2012). The potential relationship between stress and oral inflammatory infectious diseases is known for many years. A wide range of biomarkers is measurable in saliva, including hormones and their metabolites, enzymes, immunoglobulins (IgA), other proteins (eosinophil cationic protein) and DNA (Koh et al. 2007). Anxiety, depression, burnout and staff turnover are correlated with several salivary biomarkers. Chronic stress is associated with the activation of the hypothalamic-pituitary-adrenal (HPA) axis, as well as with the depression of immune function, mainly salivary IgA and lysozyme (Heinrichs et al. 2005).

Periodontal disease and its progression associated with psychosocial, financial stress and depression (Rosania et al. 2009). However, there are reports no association between stress and periodontal disease. Therefore, more research is needed to get a clearer understanding of this relationship (Rai et al. 2011). So, the aim of the current study was to determine the relationship of stress, anxiety and depression level and salivary IgA with periodontal disease.

\section{MATERIAL AND METHODS}

\section{PATIENTS}

For this case-control study, 30 patients (15 male and 15 female) referred to periodontology department, Islamic
Azad University, Dental Branch, Tehran, Iran during 2015 were included. The average age for the patients was 42-44 years old. All patients were informed about the study and signed the agreement form.

\section{STUDY PROTOCOL}

Two types of the patients were included into the study. The criteria for control group were the patients who not being periodontal disease and the case group were the patients who suffer periodontal disease. Then patients were divided into 2 experimental groups ( $n=15$ in each). Group control patients do not suffer from any periodontal disease and patients CAL up 3 millimeters or more and BOP in upper teeth (case group). The probing pocket depth (PPD), clinical attachment level (CAL), plaque index (PI), Gingival Index (GI) and bleeding on probing (BOP) examinations were done. All cases were evaluated for stress, anxiety and depression level by DASS42 test. Salivary samples were obtained using spitting method. Briefly, patients collected the oral cavity saliva for 5 minutes; collected into a sterile container ant $-20^{\circ} \mathrm{C}$ and then IgA level is determined with enzyme-linked immunosorbent assays (ELISA) detecting kits. To minimize experimental error, all experimental procedure was done at 11:00 AM until 13:00 PM.

\section{STATISTICAL ANALYSIS}

Data analyzed by repeated measure two-way analysis of variance (ANOVA) using SPSS 16.0 for Windows (SPSS, Inc., Chicago, IL, USA). For treatment showing a main effect by ANOVA, means were compared by $\kappa 2$ and Mendle-hazel tests. Data is presented as mean \pm standard deviation. $\mathrm{P}<0.05$ was considered as significant differences between treatments.

\section{RESULTS}

The demographic information of the patients included into the study is presented in the table 1 . According to the results, there was no significant difference for gender, age and educational level among the control (without periodontal disease) and case (with periodontal disease) groups.

As seen in table 2, no significant difference detected for PDD and PI indexes in control compared to the

\begin{tabular}{|c|c|c|c|c|c|c|}
\hline \multirow[b]{2}{*}{ Periodontal disease } & \multicolumn{2}{|c|}{ Gender } & \multirow[t]{2}{*}{ Age (years) } & \multicolumn{3}{|c|}{ Education level } \\
\hline & Male & Female & & Diploma $<$ & Diploma & University \\
\hline Control (n=15) & 9 & 6 & $42.4 \pm 5.4$ & 8 & 6 & 1 \\
\hline Case $(n=15)$ & 9 & 6 & $44.53 \pm 8.4$ & 3 & 10 & 2 \\
\hline$P$ value & \multicolumn{2}{|c|}{0.09} & 0.4 & & 0.8 & \\
\hline
\end{tabular}




\begin{tabular}{|c|c|c|c|}
\hline & \multicolumn{2}{|c|}{ Experimental groups } & \multirow[b]{2}{*}{$\mathrm{P}$ value } \\
\hline & Control $(n=15)$ & Case $(n=15)$ & \\
\hline PPD (mm) & $1.8 \pm 0.7$ & $2.7 \pm 0.4$ & $\mathrm{P}=0.05$ \\
\hline CAL (mm) & 0 & $3.002 \pm 0.6$ & $\mathrm{P}=0.001$ \\
\hline PI (\%) & $61.8 \pm 27.2$ & $75.6 \pm 22.9$ & $\mathrm{P}=0.4$ \\
\hline GI & $1.02 \pm 0.5$ & $2.1 \pm 0.7$ & $\mathrm{P}=0.001$ \\
\hline ВОР (\%) & $10.3 \pm 10.5$ & $20.5 \pm 10.4$ & $\mathrm{P}=0.001$ \\
\hline
\end{tabular}

case groups, $\mathrm{P}=0.05$ and $\mathrm{P}=0.4$, respectively. However, a significant difference detected for CAL $(P=0.001)$, GI $(\mathrm{P}=0.001)$ and BOP $(\mathrm{P}=0.001)$ indexes between two groups.

The correlation between stress, anxiety, depression and salivary IgA levels with periodontal disease is presented in table 3 . According to the results, there was no significant difference on stress $(\mathrm{P}=0.9)$, anxiety $(\mathrm{P}=0.3)$ and depression $(\mathrm{P}=0.1)$ in control compared to the case groups. However, a significant difference detected in salivary IgA levels in control compared to the case groups $(\mathrm{P}=0.001)$.

\section{DISCUSSION}

Periodontitis is an inflammatory disease caused by periodontopathic bacteria in the dental biofilm, leading to destruction of the tooth supporting tissues. Systemic diseases, habits, social factors, and psychological stress are considered risk factors influencing disease incidence and progression. Although psychological stress was found to be an important risk factor for periodontitis, the biologic mechanisms of its implication for disease progression is not fully elicited (Haririan et al. 2012).

In this study patients suffering from periodontal disease were not encountering higher level of stress and anxiety. People suffering periodontal disease (86.7\%) were depressed while $60 \%$ of people neither periodontal disease nor depressed. There are several factors included age, smoking, systemic diseases and psychological stress which are important risk factors for periodontitis (Ishisaka et al. 2007). In this regard, De Marco (1976) coined the term "Periodontal Emotional Stress Syndrome" for individuals with severe periodontitis who had emotional stress associated with active service in Vietnam suggesting a role of occupational stress in the progression of periodontitis.

Periodontitis patients with inadequate stress behaviors strategies were suggested to be at higher risk for severe periodontal diseases (Wimmer et al. 2002). Literature reported the relationship between stress, depression and periodontal disease are significant because they address critical areas of interest surrounding an important perio-systemic connection (Peruzzo et al. 2007). During stress, the HPA axis and sympathetic nervous system interact which leads to up-surge of the glucocorticoid and increases the susceptibility of the periodontal diseases (Bansal et al. 2014). The mediating effect of chronic stress on periodontium can be explained by diminished immune response which causes release of local neuropeptides such as substance $P$ and neurokinin A, may provide a mechanism for neural modification of inflammatory changes in the periodontium (Linden et al. 1998).

In the current study, no significant difference detected for the PDD and PI indexes in control compared to the case group while a significant difference detected for CAL, GI and BOP indexes between two groups. Shende et al. (2016) no significant differences showed on PI, probing depth, CAL among the subjects with varying levels of stress. There was no statistical significance for stress to be contributing toward the periodontal disease. Saliva testing is a novel, quick, painless, non-invasive diagnostic method for various diseases, in particular for oral diseases (Giannobile et al. 2009). There are numerous specific and nonspecific biomarkers affecting the biofilm which is a plaque formed by aggregates of proteins and

\begin{tabular}{|c|c|c|c|c|}
\hline \multirow[b]{2}{*}{ Factors } & & \multicolumn{2}{|c|}{ Experimental groups } & \multirow[b]{2}{*}{$P$ value } \\
\hline & & Control $(n=15)$ & Case $(n=15)$ & \\
\hline \multirow[b]{2}{*}{ Stress } & No & $5(33.3)$ & $4(26.6)$ & \multirow[b]{2}{*}{$\mathrm{P}=0.9$} \\
\hline & Yes & $10(66.7)$ & $11(73.4)$ & \\
\hline \multirow{2}{*}{ Anxiety } & No & $6(40)$ & $3(20)$ & \multirow{2}{*}{$\mathrm{P}=0.3$} \\
\hline & Yes & $9(60)$ & $12(80)$ & \\
\hline \multirow[b]{2}{*}{ Depression } & No & $6(40)$ & $2(13.3)$ & \multirow[b]{2}{*}{$\mathrm{P}=0.1$} \\
\hline & Yes & $9(60)$ & 13 (86.6) & \\
\hline Salivary IgA & - & $312.66+107.3$ & $207.95+57.21$ & $\mathrm{P}=0.001$ \\
\hline
\end{tabular}


micro bacterium. The major specific defense factors of saliva are the IgA, IgG, and IgM (Sindhu and Jagannathan, 2014).

The adherence of bacteria and bacterial metabolism interfered in presence of the immunoglobulins, particularly with the IgA type. Many biomarkers have been found in saliva and some of these were shown to be involved in periodontal disease (Giannobile et al. 2009). The salivary concentrations of these immunoglobulins increase in periodontitis which decreases following periodontal therapies (Sindhu and Jagannathan, 2014). As seen in this study, salivary IgA decreased in periodontal patients compared to the normal people (without periodontal disease). These results suggested a correlation exists between incidence of the periodontal disease and immunity. In condition pain, anxiety and stress, many metabolic and endocrine changes occur in the body, the most common effect of which is increased cortisol level in the blood. It plays a role in the regulation of the immune system and vascular reactions. Also known as the stress hormone, cortisol is a decisive index in stressful situations (Ismail et al. 2007). One of the likely mechanisms of such relationship is activation of the HPA axis following stressful life events, which leads to elevation of cortisol concentrations in gingival crevicular fluid, serum, and other body fluids. Leukocytic, chemotactic, polymorphonuclear leukocytes, IgG production and salivary IgA secretion are considerably declined by continued elevation in cortisol concentrations, placing the host in an immunosuppressive status, hence more vulnerable to periodontal infection and breakdown (Wong, 2008).

The IgA level measurement is a reliable method to determine the immune system function (Shah et al. 2009). Chronic stress decreases the immune system performance and suppresses the immunoglobulin production. So, the IgA levels play a role in the pathogenesis of oral mucosa and its associated clinical changes (Rabiei et al. 2012). The IgA and IgG levels Increased in patients with lichen planus (Sistig et al. 2002) and lichenoid reaction Lesions (Ghalayani et al. 2009). Also, Sato et al. (1991) studies compared the salivary IgA level in patients with different oral diseases using the ELISA method. An increase in the salivary IgA level was observed in patients with oral leukoplakia, oral lichen planus and carcinoma of the oral cavity. Higher IgA levels facilitate the antigen supply by Langerhans cells, make changes in the basal layer destruction and dispatch immune cells to the area (Nosratzehi et al. 2014). Furthermore, Salivary IgA levels influenced by mental stress and several studies have suggested a negative correlation between the levels of salivary IgA and stress (Fukui et al. 2010). Despite the mechanism for how Salivary IgA affects by mental stress is not fully elicited, it is reported Chromogranin A is an acidic glycoprotein which is released along with cat- echolamines from the adrenal medulla and the sympathetic nerve endings, and has been receiving attention as a novel stress marker in the saliva (Fukui et al. 2010).

In conclusion these results suggested a correlation exists between incidence of the periodontal disease and immunity. We think obtained results can use as information for clinical applications in human therapies. Also, we think further researches is needed to determine the direct molecular and cellular mechanism for observed data.

\section{REFERENCES}

Bansal, J., Bansal, A., Shahi, M., Kedige, S. and Narula, R. 2014 Periodontal Emotional Stress Syndrome:Review of Basic Concepts, Mechanism and Management. Open Journal of Medical Psychology, 3, 250-261.

Castro GDC, Oppermann RV, Haas AN, Winter R, Alchieri JC: 2006. Association between Psychosocial factors and Periodontitis. J Clin Periodontol 33:109-114.

De Marco, T. (1996) Periodontal Emotional Stress Syndrome. Journal of Periodontology, 47, 67-68.

Fukui M, Hinode D, Yokoyama M, Yoshioka M, Kataoka K, Ito HO. 2010 Levels of salivary stress markers in patients with anxiety about halitosis. Archives of oral biology 55 842-847.

Ghalayani P, Razavi SM, Gholami D. 2009. Comparative study of number and distribution of IgG+ cells in oral lichen planus and oral lichenoid lesions. Dent Res J (Isfahan). 6(1): $1-5$.

Giannobile WV, Beikler T, Kinney JS, Ramseier CA, Morelli T, Wong DT.2009 Saliva as a diagnostic tool for periodontal disease: Current state and future directions. Periodontol 2000 50:52-64.

Gundala R, Chava VK, Ramesh RBV.2012 Role of Stress in Periodontal Disease. Indian J Dent Adv 4(1): 763-771

Heinrichs M, Wagner D, Schoch W, et al.2005. Predicting posttraumatic stress symptoms from pretraumatic risk factors: a 2-year prospective follow-up study in firefighters. Am J Psychiatry 162:2276-86.

Ishisaka, A., Ansai, T., Soh, I., Inenaga, K., Yoshida, A., Shigeyama, C., et al. (2007) Association of Salivary Levels

Ismail SB, Kumar SK, Zain RB.2007.Oral licheplanus and lichenoid reactions: Etiopathogenesis, diagnosis, management and malignantn transformation. J Oral Sci. 49(2): 89-106.

Koh DSQ, Koh G Ch H. 2007. The use of salivary biomarkers in occupational and environmental medicine. Occup Environ Med 64:202-210.

Lakshmi Boyapati and Hom-lay Wang.2007. The role of stress in periodontal disease and wound healing. Periodontol; 44:195-210.

Linden, G.J., McKinnell, J., Shaw, C. and Lundy, F.T. (1998) Substance P and Neurokinin A in Gingival Crevicular Fluid in 
Periodontal Health and Disease. Journal of Clinical Periodontology, 24, 799-803.

Nosratzehi T, Arbabi-Kalati F, Salimi S, Honarmand E. 2014. The evaluation of psychological factor and salivary cortisol and IgA levels in patients with oral lichen planus. ZJRMS 16(7): 31-34

of Cortisol and Dehydroepiandrosterone with Periodontitis in Older Japanese Adults. Journal of Periodontology, 78, 17731767.

Peruzzo, D.C., Benatti, B.B., Ambrosano, G.M., Nogueira-Filho, G.R., Sallum, E.A., Casati, M.Z. and Nociti, F.H.(2007) A Systematic Review of Stress and Psychological Factors as Possible Risk Factors for Periodontal Disease. Journal of Periodontology, 78, 1491-1504.

Rabiei M, Sadegh-Kanjani M, Kazemnezhad-Leili E and Kohanghadam S. 2012. The comparsion between anxiety, level of salivary cortisol SIgA in oral lichen planus. J Res Dent Sci. 9(3): 125-131.

Radafshar G., Zarrabi H., Jalayer S. 2012. Relationships of Stress and Coping Styles to Periodontal Disease: A Case-Control Study Journal of Dentistry Shiraz University of Medical Sciences 13(4): 169-175.

Rai B, Kaur J, Anand SC, Jacobs R. 2011. Salivary stress markers, stress, and periodontitis: a pilot study. J Periodontol 82: 287-292.
Rosania AE, Low KG, McCormick CM, Rosania DA. 2009. Stress, depression, cortisol, and periodontal disease. J Periodontol 80: 260-266.

Sato K.1991. Enzyme-linked immunosorbent assay of SIgA in whole saliva of healthy subjects and patients with oral diseases. Bull Tokyo Med Dent Univ. 38(2): 9-18.

Shah B, Ashok L, Sujatha GP. Evalution of salivary cortisol and psychological factors in patients with lichen planus. Indian J Dent Res. 20(3): 288-92.

Shende AS, Bhatsange AG, Waghmare AS, Shiggaon LB, Mehetre VN, Meshram EP.2016. Determining the association between stress and periodontal disease: A pilot study. J Int Clin Dent Res Organ 8:111-4.

Sindhu S, Jagannathan N. Saliva: 2014 A Cutting Edge in Diagnostic Procedures. Journal of Oral Diseases. Volume Article ID 168584, 8 pages. http://dx.doi.org/10.1155/2014/168584

Sistig S, Vucicevic-Boras V, Lukac J and Kusic Z.2002. Salivary IgA and IgG subclasses in oral mucosal diseases. Oral Dis. 8(6): 282-6.

Wimmer, G., Janda, M., Wieselmann-Penkner, K., Jakse, N., Polansky, R. and Pertl, C. (2002) Coping with Stress: Its Influence on Periodontal Disease. Journal of Periodontology, 73, 1343-351.

Wong D.2008. Salivary diagnostics. 1st ed. New Jersey: WileyBlackwell; 2008: 37-59. 